regression assessed factors associated with number of GVclades. Generalized estimating equations population-averaged models assessed factors associated with each GV-clade. Models accounted for repeated measures.

Results 369 specimens from 101 women were analysed. GV was detected in 181 specimens, and most GV-positive specimens had multiple clades present $(n=119 / 181,66 \%)$. Detection of multiple GV-clades was associated with smoking (adjusted relative risk ratio [RRR]:2.52; 95\%CI:1.25,5.07), increased lifetime female sex partners (FSP; adjRRR:2.43; 95\%CI:1.09,5.38), and a NS=4-6 (intermediate microbiota) or NS=7-10 (Nugent BV) relative to no clades. GV4 was the most prevalent clade $(\mathrm{n}=136 / 369 ; 37 \% ; 95 \%$ CI: 32,42\%), followed by GV1 ( $\mathrm{n}=116 / 369 ; 31 \% ; 95 \% \mathrm{CI}: 27,36 \%)$ and GV2 (n=76/369; 21\%; 95\% CI: 17,25\%). GV3 was uncommon $(n=17 / 369 ; 5 \% ; 95 \%$ CI:3,7\%). GV1 was associated with a NS=7-10 (adjusted odds ratio[AOR]:3.87; 95\% CI:1.75,8.56), smoking (AOR:2.74; 95\%CI:1.28,5.87) and report of any sexual partners (AOR:3.41; 95\%CI:1.18,9.86). GV2 was associated with $\mathrm{NS}=4-6$ (AOR:3.28; 95\% CI:1.00,10.77), sharing of sex-toys (AOR:2.30; 95\% CI:1.05,5.04) and recent male sex partners (AOR:6.58; 95\% $\mathrm{CI}: 2.02,21.40)$. GV4 presence was associated with increased lifetime FSPs (AOR:3.17; 95\%CI:1.25,5.07).

Conclusion GV1 and presence of multiple GV-clades was associated with Nugent BV in WSW, whereas GV2 was associated with intermediate microbiota. Individual GV-clades were associated with a range of differing sexual behaviours in adjusted analyses. These associations are of uncertain importance, but do provide support for exchange of $G V$-clades between sexual partners.

Disclosure No significant relationships.

\section{P365 MICROBIAL RISK FACTORS FOR ACQUISITION OF SYMPTOMATIC BACTERIAL VAGINOSIS (BV)}

${ }^{1}$ May Beamer*, ${ }^{2}$ Leslie Meyn, ${ }^{1}$ Melinda Petrina, ${ }^{1}$ Lisa Cosentino, ${ }^{1}$ Hilary Avolia, ${ }^{1}$ Michele Austin, ${ }^{1}$ Allison Demarco, ${ }^{1}$ Victoria Gould, ${ }^{2}$ Sharon Hillier. ${ }^{1}$ Magee-Womens Research Institute, Pittsburgh, USA; ${ }^{2}$ University of Pittsburgh and Magee-Womens Research Institute, Obstetrics, Gynecology and Reproductive Sciences, Pittsburgh, USA

\subsection{6/sextrans-2019-sti.467}

Background Vaginal dysbiosis is common among women of reproductive age but many women having vaginal dysbiosis do not develop symptomatic BV requiring treatment. Our objective was to identify microbiota using quantitative PCR (qPCR) which were associated with acquisition of symptomatic BV.

Methods In this secondary analysis of a vaccine trial, 440 healthy asymptomatic sexually active women aged 18-40 had vaginal swabs collected at baseline and returned at 1, 2 and 4 months. Eleven vaginal microbes at baseline were identified using qPCR (five species of Lactobacillus (crispatus, vaginalis, jensenii, gasseri, iners), three species of Prevotella (bivia, timonensis, amnii), Atopobium vaginae (AVAG), Gardnerella vaginalis (GVAG) and Megasphaera phylotype I (MEGA). Time to first acquisition of symptomatic BV was based on self-report of antibiotic treatment for BV. Cox proportional hazards models were used to evaluate the association of microbiota with BV acquisition.

Results Thirty-five women developed symptomatic BV over 130.7 person-years (PY) of follow-up for an overall rate of 27/100PY. Women who acquired BV had a lower baseline prevalence of $L$. crispatus than those who did not $(31.4 \%$ vs
$57.8 \% ; P=0.012)$, and a higher prevalence and log concentration of GVAG (97.1\% vs $76.0 \%)$, AVAG $(77.1 \%$ vs $43.7 \%)$, MEGA (65.7\% vs $23.2 \%)$, P. timonensis $(80.0 \%$ vs $61.7 \%)$, and $P$. amnii $(51.4 \%$ vs $20.5 \%),(P \leq 0.001)$. In multivariable analyses, women who had GVAG, AVAG, and MEGA at baseline had a significantly higher BV acquisition rate (74/100 PY, hazard ratio $=21.2 ; 95 \%$ confidence interval: $2.8-158.8)$ compared to women who had none of these microorganisms (rate $=4 / 100$ PY). Women who had only one (BV rate $=16 / 100$ $\mathrm{PY})$ or two (BV rate $=14 / 100 \mathrm{PY}$ ) of the three microbes detected at baseline were not more likely to acquire BV $(\mathrm{P} \geq 0.14)$.

Conclusion Although many individual bacteria contribute to the dysbiotic communities associated with $\mathrm{BV}$, the combination of GVAG, AVAG, and MEGA rather than presence of lactobacilli predicts symptomatic BV.

Disclosure No significant relationships.

\section{P366 AN EVALUATION OF THE BD MAXTM VAGINAL PANEL COMPARED TO THE NUGENT SCORE FOR DIAGNOSIS OF BACTERIAL VAGINOSIS (BV)}

${ }^{1}$ Lisa Cosentino*, ${ }^{1}$ Michele Austin, ${ }^{1}$ May Beamer, ${ }^{2}$ Sharon Hillier. ${ }^{1}$ Magee-Womens Research Institute, Pittsburgh, USA; ${ }^{2}$ University of Pittsburgh and Magee-Womens Research Institute, Obstetrics, Gynecology and Reproductive Sciences, Pittsburgh, USA

\subsection{6/sextrans-2019-sti.468}

Background The Nugent criteria have been used as a comparator to Amsel criteria for diagnosis of $\mathrm{BV}$. The $\mathrm{BD} \mathrm{MAX}^{\mathrm{TM}}$ Vaginal Panel (MVP) received market authorization by the FDA as a molecular test to aid in the diagnosis of vaginitis/ vaginosis. The assay detects Lactobacillus crispatus, L. jensenii, Gardnerella vaginalis, Atopobium vaginae, Megasphaera-1 and BVAB-2, utilizing a semi-quantitative algorithm. Our objective was to evaluate agreement between Nugent score (NS) and MVP and to identify factors contributing to the discordance between methods.

Methods 303 women aged $18-40$ presenting with a complaint of vaginitis symptoms provided informed consent and were enrolled at 8 community practice sites affiliated with a single medical system. Clinicians evaluated study participants per local practice and 5 additional vaginal swabs were collected. Vaginal swabs were evaluated by the MVP, plus NS $(\geq 6)$ for diagnosis of BV. Quantitative PCR (qPCR) was used to evaluate vaginal microbiota in the samples yielding discordant results.

Results Of the 290 evaluable samples, the level of agreement between NS and MVP was $88 \%$ with 36 discordant results. There were 6 Nugent $0-3$ and 13 NS 4-6 that were BV positive by MVP. Of these 19, the median concentration of $L$. iners by qPCR was $\log$ concentration 7.3 , A. vaginae was 6.7 and $G$. vaginalis was 7.5 . Of the 17 positive for BV by NS (median NS 8) but negative by MVP, the median concentration of $G$. vaginalis was 7.5 but A. vaginae and L. crispatus had medians of 0 by qPCR.

Conclusion Nugent score and MVP had high levels of agreement for diagnosis of BV. Because L. iners is indistinguishable from L. crispatus and L. jensenii by Gram stain, high concentrations of this microorganism contribute to lower NS and substantially contributes to the NS-/MVP+ discordance. The primary source of discordance for NS+/MVP- samples is lack of $A$. vaginae.

Disclosure No significant relationships. 